\title{
Convergence of Vectorial Continued Fractions Related to the Spectral Seminorm
}

\author{
M. Hemdaoui and M. Amzil \\ Laboratoire G.A.F.O, Département de Mathmatiques \& Informatique, Faculté des Sciences, \\ Université Mohamed Premier, Oujda, Morocco \\ Correspondence should be addressed to M. Hemdaoui, mhemdaoui@yahoo.fr \\ Received 07 February 2008; Accepted 16 April 2008 \\ Recommended by Charles Chidume \\ We show that the spectral seminorm is useful to study convergence or divergence of vectorial \\ continued fractions in Banach algebras because such convergence or divergence is related to a \\ spectral property. \\ Copyright (c) 2008 M. Hemdaoui and M. Amzil. This is an open access article distributed under \\ the Creative Commons Attribution License, which permits unrestricted use, distribution, and \\ reproduction in any medium, provided the original work is properly cited.
}

\section{Introduction}

Let $\mathcal{A}$ be a unital complex Banach algebra. We denote by $e$ the unit element of $\mathcal{A}$. $\|\cdot\|$ is the norm of $\mathcal{A}$. For $a \in \mathcal{A}, \sigma(a)$, and $\rho(a)$ denote, respectively, the spectrum and the spectral seminorm of $a$.

A formal vectorial continued fraction is an expression of the form

$$
y_{0}=b_{0}+a_{1} \cdot\left(b_{1}+a_{2} \cdot\left(b_{2}+\cdots\right)^{-1}\right)^{-1},
$$

where $\left(a_{n}\right)_{n \geq 1}$ and $\left(b_{n}\right)_{n \geq 1}$ are two sequences of elements in $\mathcal{A}$.

In order to discuss convergence or divergence of the vectorial continued fraction (1.1), we associate a sequence $\left(s_{n}\right)_{n \geq 0}$ (called sequence of $n$th approximants) defined by:

$$
\begin{aligned}
s_{0}= & b_{0} \\
s_{1}= & b_{0}+a_{1} \cdot b_{1}^{-1}, \\
s_{2}= & b_{0}+a_{1} \cdot\left(b_{1}+a_{2} \cdot b_{2}^{-1}\right)^{-1}, \\
\vdots & \vdots \\
s_{n}= & b_{0}+a_{1} \cdot\left(b_{1}+a_{2} \cdot\left(b_{2}+\cdots+a_{n-1} \cdot\left(a_{n-1}+a_{n} \cdot b_{n}^{-1}\right)^{-1}\right)^{-1}\right)^{-1},
\end{aligned}
$$


By induction, it can be shown that

$$
s_{n}=b_{0}+p_{n} \cdot q_{n}^{-1},
$$

where the expressions $p_{n}$ and $q_{n}$ are determined from recurrence relations

$$
\begin{aligned}
& p_{n+1}=p_{n} \cdot b_{n+1}+p_{n-1} \cdot a_{n+1}, \\
& q_{n+1}=q_{n} \cdot b_{n+1}+q_{n-1} \cdot a_{n+1},
\end{aligned}
$$

with initial conditions:

$$
\begin{array}{ll}
p_{0}=0, & p_{1}=a_{1} . \\
q_{0}=e, & q_{1}=b_{1} .
\end{array}
$$

$p_{n}$ and $q_{n}$ are respectively called $n$th numerator and $n$th denominator of (1.1).

Now, consider the following example.

Let $a$ be a nonnull quasinilpotent element in $\mathcal{A}$. Consider the vectorial continued fraction defined by

$$
\left[(e+a)+\left[\left(\frac{1}{4} e+a\right)+\left[\left(\frac{1}{9} e+a\right)+\cdots+\left[\left(\frac{1}{n^{2}} e+a\right)+\cdots\right]^{-1}\right]^{-1}\right]^{-1}\right]^{-1},
$$

where for each positive integer $n>0$, we have

$$
b_{n}=\frac{1}{n^{2}} \cdot e+a
$$

So,

$$
\left\|b_{n}\right\|=\left\|a+\frac{1}{n^{2}} e\right\| \geq\left|\|a\|-\frac{1}{n^{2}}\right| .
$$

Therefore, the series $\sum_{n=1}^{\infty}\left\|b_{n}\right\|$ diverges.

By Fair [1, Theorem 2.2], we cannot ensure convergence or divergence of the vectorial continued fraction (1.6). But, if we apply the spectral seminorm to (1.7), we get

$$
\rho\left(b_{n}\right) \leq \frac{1}{n^{2}}+\rho(a)=\frac{1}{n^{2}} .
$$

So, the series $\sum_{n=1}^{\infty} \rho\left(b_{n}\right)$ converges. From Theorem 2.5 in Section 2 below, the vectorial continued fraction (1.6) diverges according to the spectral seminorm so it diverges also according to the norm because the spectral seminorm $\rho$ satisfies

$$
\rho(x) \leq\|x\|, \quad \forall x \in \mathcal{A} .
$$

In Section 3, we give another example of a vectorial continued fraction that converges according to the spectral seminorm and diverges according to the norm algebra.

From the simple and particular example above and the example in Section 3, we see that to study convergence or divergence of vectorial continued fractions we can use the spectral seminorm of the algebra to include a large class of vectorial continued fractions.

First, we start by determining necessary conditions upon $a_{n}$ and $b_{n}$ to ensure the convergence.

Next, we give sufficient conditions to have the convergence. 


\section{Convergence of vectorial continued fractions}

In this section, we discuss some conditions upon the elements $a_{n}$ and $b_{n}$ of the vectorial continued fraction (1.1) (with $b_{0}=0$ ) which are necessary to ensure the convergence.

Definition 2.1. The vectorial continued fraction (1.1) converges if $q_{n}^{-1}$ exists starting from a certain rank $N$, and the sequence of $n$th approximants $s_{n}$ converges. Otherwise, the vectorial continued fraction (1.1) diverges.

For future use, we record the following theorem due to P. Wynn.

Theorem $2.2([2])$. For all $n \in \mathbb{N}$, we have

$$
\begin{aligned}
s_{n+1}-s_{n} & =p_{n+1} \cdot q_{n+1}^{-1}-p_{n} \cdot q_{n}^{-1} \\
& =(-1)^{n} a_{1} b_{1}^{-1} q_{0} a_{2} q_{2}^{-1} q_{1} a_{3} q_{3}^{-1} q_{2} a_{4} q_{4}^{-1} \cdots q_{n-2} a_{n} q_{n}^{-1} q_{n-1} a_{n+1} q_{n+1}^{-1} .
\end{aligned}
$$

Remark 2.3. In the commutative case, Theorem 2.2 above becomes as follows.

For all $n \in \mathbb{N}$, one has

$$
s_{n+1}-s_{n}=(-1)^{n}\left(\prod_{i=1}^{i=n+1} a_{i}\right) \cdot q_{n+1}^{-1} \cdot q_{n}^{-1} .
$$

Since convergence or divergence of the vectorial continued fraction (1.1) is not affected by the value of the additive term $b_{0}$, we omit it from subsequent discussion (i.e., $b_{0}=0$ ).

Now, we give a proposition that extends a result due to Wall [3] in the case of scalar continued fractions.

Proposition 2.4. The vectorial continued fraction (1.1) where its terms are commuting elements in $A$ diverges, if its odd partial denominators $b_{2 n+1}$ are all quasinilpotent elements in $\mathcal{A}$.

Proof. In fact, from relation (1.5) above, we have $q_{1}=b_{1}$. So $\rho\left(q_{1}\right)=\rho\left(b_{1}\right)=0$.

Since coefficients of (1.1) are commuting elements in $\mathcal{A}$, it is easy to show that for all positive integers $n$ and $m$, we have

$$
a_{m} \cdot q_{n}=q_{n} \cdot a_{m} ; \quad b_{m} \cdot q_{n}=q_{n} \cdot b_{m} .
$$

So,

$$
\rho\left(a_{m} \cdot q_{n}\right) \leq \rho\left(a_{m}\right) \cdot \rho\left(q_{n}\right) ; \quad \rho\left(b_{m} \cdot q_{n}\right) \leq \rho\left(b_{m}\right) \cdot \sigma\left(q_{n}\right) .
$$

Now, suppose that for $n \geq 1, \rho\left(q_{2 n-1}\right)=0$.

From relations (1.4) and (2.4), we have

$$
\rho\left(q_{2 n+1}\right) \leq \rho\left(q_{2 n}\right) \cdot \rho\left(b_{2 n+1}\right)+\rho\left(q_{2 n-1}\right) \cdot \rho\left(a_{2 n+1}\right) .
$$

Then, $\rho\left(q_{2 n+1}\right)=0$, consequently

$$
\forall n \geq 0 ; \quad \rho\left(q_{2 n+1}\right)=0 .
$$

So infinitely many denominators $q_{n}$ are not invertible.

The vectorial continued fraction (1.1) diverges. 
Theorem 2.5 below gives a necessary condition for convergence according to the spectral seminorm. This result is an extension of von Koch Theorem [4], concerning the scalar case. A similar theorem was given by Fair [1] for vectorial continued fractions according to the norm convergence.

Theorem 2.5. Let $a_{n}=e$, for all $n \geq 1$, and $b_{n}$ be a sequence of commuting elements in $\mathcal{A}$. If the vectorial continued fraction (1.1) converges according to spectral seminorm, then, the series $\sum_{n=1}^{\infty} \rho\left(b_{n}\right)$ diverges.

Proof. Suppose $\sum_{n=1}^{\infty} \rho\left(b_{n}\right)$ is a converging series, and there exists a positive integer $N$ such that $q_{n}^{-1}$ exists, for all $n \geq N$.

By an induction argument, it is easy to show that for all $n \in \mathbb{N}$, we have

$$
\rho\left(q_{2 n}-e\right) \leq \exp \left(K_{2 n}\right)-1, \quad \rho\left(q_{2 n+1}\right) \leq \exp \left(K_{2 n+1}\right),
$$

where $K_{0}=0$ and $K_{n}=\sum_{k=1}^{n} \rho\left(b_{k}\right)$; for all $n \geq 1$.

Since for all positive integer $n, a_{n}=e$, and all $b_{n}$ are commuting elements in $\mathcal{A}$, from Remark 2.3 above, we have

$$
d_{n}=s_{2 n+1}-s_{2 n}=q_{2 n+1}^{-1} \cdot q_{2 n}^{-1}, \quad \forall n \geq E\left[\frac{N}{2}\right]+1
$$

So,

$$
\rho\left(d_{n}\right) \geq\left[\rho\left(d_{n}^{-1}\right)\right]^{-1}=\left[\rho\left(q_{2 n+1} \cdot q_{2 n}\right)\right]^{-1}, \quad \forall n \geq E\left[\frac{N}{2}\right]+1
$$

Then,

$$
\rho\left(d_{n}\right) \geq\left[\rho\left(q_{2 n+1}\right)\right]^{-1} \cdot\left[\rho\left(q_{2 n}\right)\right]^{-1}, \quad \forall n \geq E\left[\frac{N}{2}\right]+1
$$

From this preceding,

$$
\rho\left(d_{n}\right) \geq \frac{1}{\exp \left(K_{2 n+1}\right)} \cdot \frac{1}{\exp \left(K_{2 n}\right)-1} \geq \frac{1}{\exp \left(2 K_{2 n+1}\right)} \geq \frac{1}{\exp (2 K)}>0,
$$

where $K=\sum_{n=1}^{\infty} \rho\left(b_{n}\right)$.

So, the sequence $\left(s_{n}\right)_{n \geq 0}$ is not a $\rho$-Cauchy sequence in $\mathcal{A}$.

Remark 2.6. In a Banach algebra $\mathcal{A}$ if $\rho$ denotes the spectral seminorm in $\mathcal{A}$ it is not a multiplicative seminorm in general.

Consider the vectorial subspace of $\mathcal{A}$ defined by $\operatorname{Ker}(\rho)=\{x \in \mathcal{A} \mid \rho(x)=0\}$. The quotient vectorial space $\mathcal{A} / \operatorname{Ker}(\rho)$ becomes a normed vectorial space with norm defined by $\dot{\rho}(\dot{x})=\rho(x), x \in \dot{x}$. " $\dot{x}$ denotes the class of $x$ modulo $\operatorname{Ker}(\rho)$."

Generally, the normed vectorial space $\mathcal{A} / \operatorname{Ker}(\rho)$ is not complete. Its complete normed vectorial space is $\widehat{\mathcal{A} / \operatorname{Ker}(\rho)}$ witch is a Banach space. So, $\rho$-Cauchy sequences in $(\mathcal{A}, \rho)$ converge in the Banach space $\widehat{\mathbb{A} / \operatorname{Ker}(\rho)}$. 
Remark 2.7. Whenever $\mathcal{A}$ is commutative, the vectorial continued fraction (1.1) diverges, if for one character $\psi$, the series $\sum_{n \geq 1}\left|\psi\left(b_{n}\right)\right|$ converges.

Lemma 2.8. Let $\left(u_{n}\right)_{n}$ be a sequence of commuting elements in $\mathcal{A}$.

If the series $\sum_{n \geq 1} \rho\left(u_{n}\right)$ converges, then, there exists a positive integer $N \geq 1$ such that for every positive integer $k \geq 1$, the finite product $\prod_{p=1}^{k}\left(e+u_{N+p}\right)$ is invertible and $\rho$-bounded and its inverse is also $\rho$-bounded.

Proof. Since the series $\sum_{n \geq 1} \rho\left(u_{n}\right)$ converges, therefore, there exists a positive integer $N \geq 1$ such that

$$
\rho\left(u_{n}\right)<1 ; \quad \forall n \geq N
$$

Hence, for $k \geq 1$ the product $\prod_{p=1}^{k}\left(e+u_{N+p}\right)$ is invertible as finite product of invertible elements. We have

$$
\rho\left(\prod_{p=1}^{k}\left(e+u_{N+p}\right)\right) \leq \prod_{p=1}^{k}\left(1+\rho\left(u_{N+p}\right)\right) \leq \prod_{p=1}^{+\infty}\left(1+\rho\left(u_{N+p}\right)\right) .
$$

But

$$
\left(\prod_{p=1}^{k}\left(e+u_{N+p}\right)\right)^{-1}=\prod_{p=1}^{k}\left(e+u_{N+p}\right)^{-1}=\prod_{p=1}^{k} \sum_{n=0}^{+\infty}(-1)^{n} u_{N+p}^{n} .
$$

Hence,

$$
\begin{aligned}
& \rho\left(\left(\prod_{p=1}^{k}\left(e+u_{N+p}\right)\right)^{-1}\right) \\
& \quad \leq \prod_{p=1}^{k} \sum_{n=0}^{+\infty} \rho^{n}\left(u_{N+p}\right)=\prod_{p=1}^{k} \frac{1}{1-\rho\left(u_{N+p}\right)}=\frac{1}{\prod_{p=1}^{k}\left(1-\rho\left(u_{N+p}\right)\right)} \leq \frac{1}{\prod_{p=1}^{+\infty}\left(1-\rho\left(u_{N+p}\right)\right)} .
\end{aligned}
$$

Theorem 2.9. Let in the vectorial continued fraction (1.1) $a_{n}=e$ for all $n \geq 1$ and $\left(b_{n}\right)_{n \in \mathbb{N}}$ be $a$ sequence of commuting elements in $A$. If both series

$$
\sum_{n \geq 0} \rho\left(b_{2 p+1}\right), \quad \sum_{n \geq 0} \rho\left(b_{2 p+1}\right) \cdot \rho^{2}\left(b_{2 p}\right)
$$

converge, then, the vectorial continued fraction (1.1) diverges.

Proof of Theorem 2.9. Since both series $\sum_{n \geq 0} \rho\left(b_{2 p+1}\right)$ and $\sum_{n \geq 0} \rho\left(b_{2 p+1}\right) \cdot \rho^{2}\left(b_{2 p}\right)$ converge, it follows that the series $\sum_{n \geq 0} \rho\left(b_{2 p+1}\right) \cdot \rho\left(b_{2 p}\right)$ converges too.

Therefore, from Lemma 2.8 above, there exists a positive integer $N \geq 1$ such that for $k \geq 1$, the quantity $\theta_{k}=\prod_{p=1}^{k}\left(1+b_{2 N+2 p+1} \cdot b_{2 N+2 p}\right)$ is invertible. 
Now, consider the vectorial continued fraction

$$
\left(c_{1}+\left(c_{2}+\left(c_{3}+\cdots\right)^{-1}\right)^{-1}\right)^{-1}
$$

where

$$
c_{2 k}=b_{2 N+2 k+1} \cdot \theta_{k-1}^{-1} \cdot \theta_{k}^{-1}, \quad c_{2 k-1}=-b_{2 N+2 k+1} \cdot b_{2 N+2 k}^{2} \cdot \theta_{k-1} \cdot \theta_{k}, \quad k=1,2, \ldots
$$

We will suppose that $q_{n}^{-1}$ exists for all $n \geq N$ (otherwise, from Definition 2.1, the vectorial continued fraction (1.1) diverges).

Before continuing the proof, we give the following lemma that will be used later.

Lemma 2.10. For all positive integers $k \geq 1$, consider the quantities

$$
\begin{array}{cl}
U_{2 k}=p_{2 N+2 k+1} \cdot \theta_{k}^{-1}, \quad & V_{2 k}=q_{2 N+2 k+1} \cdot \theta_{k}^{-1}, \\
U_{2 k+1}=p_{2 N+2 k} \cdot \theta_{k}, \quad & V_{2 k+1}=q_{2 N+2 k} \cdot \theta_{k}, \\
c_{2 k}=b_{2 N+2 k+1} \cdot \theta_{k-1}^{-1} \cdot \theta_{k}^{-1}, \quad c_{2 k-1}=-b_{2 N+2 k+1} \cdot b_{2 N+2 k}^{2} \cdot \theta_{k-1} \cdot \theta_{k}, \\
k=1,2, \ldots, \quad\left(\theta_{0}=e\right) .
\end{array}
$$

Then,

$$
\begin{gathered}
U_{k}=U_{k-1} \cdot c_{k}+U_{k-2}, \\
V_{k}=V_{k-1} \cdot c_{k}+V_{k-2}, \quad \forall k \geq 2 .
\end{gathered}
$$

This lemma is proved by the same argument given by Wall [3, Lemma 6.1] for scalar continued fractions.

Lemma 2.10 shows that $U_{n}$ and $V_{n}$ are respectively the $n$th numerator and $n$th denominator of the vectorial continued fraction (2.17).

Since both series $\sum_{n \geq 0} \rho\left(b_{2 p+1}\right), \sum_{n \geq 0} \rho\left(b_{2 p+1}\right) \cdot \rho\left(b_{2 p}\right)^{2}$ converge and from Lemma 2.8 above $\theta_{k}$ and $\theta_{k}^{-1}$ are bounded, we conclude that the series $\sum_{k \geq 1} \rho\left(c_{k}\right)$ converges.

Then, it follows as in the proof of Theorem 2.5, that the vectorial continued fraction (2.17) diverges and

$$
\rho\left(U_{2 k+1} \cdot V_{2 k+1}^{-1}-U_{2 k} \cdot V_{2 k}^{-1}\right)=\rho\left(p_{2 N+2 k} \cdot q_{2 N+2 k}^{-1}-p_{2 N+2 k+1} \cdot q_{2 N+2 k+1}^{-1}\right) \geq \exp \left(2 \sum_{k=1}^{+\infty} \rho\left(c_{k}\right)\right)>0 .
$$

So,

$$
\rho\left(s_{2 N+2 k+1}-s_{2 N+2 k}\right) \geq \exp \left(2 \sum_{k=1}^{+\infty} \rho\left(c_{k}\right)\right)>0, \quad \forall k \geq 0 .
$$

This shows that the sequence of $n$th approximants $\left(s_{n}\right)_{n \geq 1}$ is not a $\rho$-Cauchy sequence in $\mathcal{A}$. 
Now, we state Theorem 2.13 to give a sufficient condition to have convergence of the vectorial continued fraction (1.1).

A similar theorem was given by Peng and Hessel [5], to study convergence of the vectorial continued fraction (1.1) in norm where for each positive integer $n, a_{n}=e$.

Before stating the proof of Theorem 2.13, we give the following lemmas.

Lemma 2.11. Let $b$ and $c$ be two commuting elements in $A$ such that the spectrum of $b^{-1} \cdot c$ is satisfied, $\sigma\left(b^{-1} \cdot c\right) \subset B(0,1)$. Then, the element $b+c$ is invertible and its inverse satisfies $\rho\left((b+c)^{-1}\right) \leq$ $\rho\left(b^{-1}\right) /\left(1-\rho\left(b^{-1} \cdot c\right)\right)$.

Proof. Since $\sigma\left(b^{-1} \cdot c\right) \subset B(0,1)$, we have $\rho\left(b^{-1} \cdot c\right)<1$. So the element $b+c$ is invertible in $\mathcal{A}$. Its inverse is

$$
(b+c)^{-1}=b^{-1}\left(e+b^{-1} \cdot c\right)^{-1}=b^{-1} \cdot \sum_{n=0}^{\infty}(-1)^{n}\left(b^{-1} \cdot c\right)^{n}
$$

So,

$$
\rho\left((b+c)^{-1}\right) \leq \rho\left(b^{-1}\right) \cdot \sum_{n=0}^{\infty} \rho^{n}\left(b^{-1} \cdot c\right)=\frac{\rho\left(b^{-1}\right)}{1-\rho\left(b^{-1} \cdot c\right)} .
$$

Lemma 2.12. Let $\epsilon \in] 0,1\left[,\left(a_{n}\right)_{n \in \mathbb{N}}\right.$ and $\left(b_{n}\right)_{n \in \mathbb{N}}$ be two sequences of elements in $\mathcal{A}$ such that for each positive integer $n \geq 1$, the spectra of $a_{n} \cdot b_{n}^{-1}$ and $b_{n}^{-1}$ lie in the open ball $B(0,(1 / 2) \epsilon)$. Then, for each positive integer $n \geq 1, q_{n}^{-1}$ exists and $\rho\left(q_{n}^{-1} \cdot q_{n-1}\right)<\epsilon$.

Where $q_{n}$ is the $n$th denominator of the vectorial continued fraction (1.1).

Proof. From recurrence relation (1.5) above, we have

$$
q_{0}=e, \quad q_{1}=b_{1},
$$

then, $q_{1}^{-1}=b_{1}^{-1}$ and $\rho\left(q_{1}^{-1} \cdot q_{0}\right)=\rho\left(b_{1}^{-1}\right) \leq(1 / 2) \epsilon<\epsilon$.

Now, suppose that for $n \geq 2, q_{n-1}^{-1}$ exists and $\rho\left(q_{n-1}^{-1} \cdot q_{n-2}\right)<\epsilon$.

Then, from recurrence relation (1.4) above, we have

$$
q_{n}=q_{n-1} \cdot b_{n}+q_{n-2} \cdot a_{n}=q_{n-1} \cdot\left(b_{n}+q_{n-1}^{-1} \cdot q_{n-2} \cdot a_{n}\right) .
$$

Put

$$
c=q_{n-1}^{-1} \cdot q_{n-2} \cdot a_{n}, \quad b=b_{n} .
$$

Appling Lemma 2.11, we have

$$
\rho\left(b^{-1} \cdot c\right) \leq \rho\left(q_{n-1}^{-1} \cdot q_{n-2}\right) \cdot \rho\left(b_{n}^{-1} \cdot a_{n}\right)<\frac{1}{2} \epsilon .
$$

So $\left(b_{n}+q_{n-1}^{-1} \cdot q_{n-2} \cdot a_{n}\right)$ is invertible and its inverse satisfies

$$
\rho\left(\left(b_{n}+q_{n-1}^{-1} \cdot q_{n-2} \cdot a_{n}\right)^{-1}\right)<\frac{(1 / 2) \epsilon}{1-(1 / 2) \epsilon}<\frac{(1 / 2) \epsilon}{1-1 / 2}<\epsilon .
$$

Therefore, $q_{n}^{-1}$ exists. So, for all $n \geq 0, q_{n}$ is invertible and $\rho\left(q_{n}^{-1} \cdot q_{n-1}\right)<\epsilon$. 
Theorem 2.13. Let $\epsilon \in] 0,1\left[, a_{n}\right.$ and $b_{n}$ be commuting terms of the vectorial continued fraction (1.1) such that for each positive integer $n \geq 1$, the spectra of $a_{n} \cdot b_{n}^{-1}$ and $b_{n}^{-1}$ lie in the open ball $B(0,(1 / 2) \epsilon)$. Then, the vectorial continued fraction (1.1) converges.

Proof of Theorem 2.13. For positive integers $n \geq 1$ and $m \geq 1$, we introduce the finite vectorial continued fraction

$$
s_{m}^{(n)}=a_{n+1} \cdot\left(b_{n+1}+a_{n+2} \cdot\left(b_{n+2}+\cdots+a_{n+m-1} \cdot\left(b_{n+m-1}+a_{n+m} \cdot b_{n+m}^{-1}\right)^{-1}\right)^{-1}\right)^{-1}
$$

with initial conditions

$$
s_{0}^{(n)}=0, \quad s_{m}^{(0)}=s_{m}
$$

where $s_{m}$ is the $m$ th approximant of the continued fraction (1.1).

It is easily shown from (2.30) that

$$
s_{m}^{(n)}=a_{n+1} \cdot\left(b_{n+1}+s_{m-1}^{(n+1)}\right)^{-1} .
$$

By the repeated use of Lemma 2.11 in each iteration in (2.30) for every $n \geq 1$ and every $m \geq 1$, we can show that for each $n$ and $m,\left(b_{n+1}+s_{m-1}^{(n+1)}\right)^{-1}$ exists and

$$
\rho\left(s_{m}^{(n)}\right)<\epsilon
$$

We have

$$
\left(b_{n+1}+s_{m}^{(n+1)}\right)^{-1}-\left(b_{n+1}+s_{m-1}^{(n+1)}\right)^{-1}=\left(b_{n+1}+s_{m}^{(n+1)}\right)^{-1} \cdot\left[s_{m-1}^{(n+1)}-s_{m}^{(n+1)}\right] \cdot\left(b_{n+1}+s_{m-1}^{(n+1)}\right)^{-1} .
$$

Thus, from relations (2.32) and (2.34), we have

$$
\begin{aligned}
s_{m+1}^{(n)}-s_{m}^{(n)} & =a_{n+1} \cdot\left(\left(b_{n+1}+s_{m}^{(n+1)}\right)^{-1}-\left(b_{n+1}+s_{m-1}^{(n+1)}\right)^{-1}\right) \\
& =a_{n+1} \cdot\left(b_{n+1}+s_{m}^{(n+1)}\right)^{-1} \cdot\left(s_{m-1}^{(n+1)}-s_{m}^{(n+1)}\right) \cdot\left(b_{n+1}+s_{m-1}^{(n+1)}\right)^{-1} \\
& =a_{n+1} \cdot b_{n+1}^{-2} \cdot K_{m} \cdot\left(s_{m}^{(n+1)}-s_{m-1}^{(n+1)}\right) \cdot K_{m-1}
\end{aligned}
$$

where $K_{m}=\left(e+b_{n+1}^{-1} \cdot s_{m}^{(n+1)}\right)^{-1}$, for $m \in \mathbb{N}^{*}$.

Then,

$$
\rho\left(s_{m+1}^{(n)}-s_{m}^{(n)}\right) \leq \rho\left(a_{n+1} \cdot b_{n+1}^{-1}\right) \cdot \rho\left(b_{n+1}^{-1}\right) \cdot \rho\left(K_{m}\right) \cdot \rho\left(s_{m}^{(n+1)}-s_{m-1}^{(n+1)}\right) \cdot \rho\left(K_{m-1}\right) .
$$

Since from $(2.33) \rho\left(b_{n+1}^{-1} \cdot s_{m}^{(n+1)}\right) \leq \rho\left(b_{n+1}^{-1}\right) \cdot \rho\left(s_{m}^{(n+1)}\right) \leq(1 / 2) \epsilon^{2}<1 / 2$, then, using Lemma 2.11,

$$
\rho\left(K_{m}\right) \leq \frac{1}{1-\rho\left(b_{n+1}^{-1} \cdot s_{m}^{(n+1)}\right)}<2, \quad \text { for } m \in \mathbb{N}^{*},
$$

we have $\rho\left(a_{n+1} \cdot b_{n+1}^{-1}\right) \leq(1 / 2) \epsilon$ and $\rho\left(b_{n+1}^{-1}\right) \leq(1 / 2) \epsilon$. 
Then,

$$
\rho\left(s_{m+1}^{(n)}-s_{m}^{(n)}\right) \leq \epsilon^{2} \cdot \rho\left(s_{m}^{(n+1)}-s_{m-1}^{(n+1)}\right) .
$$

Gradually, we get

$$
\rho\left(s_{m+1}^{(n)}-s_{m}^{(n)}\right) \leq \epsilon^{2 m} \cdot \rho\left(s_{1}^{(n+m)}-s_{0}^{(n+m)}\right) .
$$

Besides, we have $s_{0}^{(n+m)}=0$ and $s_{1}^{n+m}=a_{n+m+1} \cdot b_{n+m+1}^{-1}$.

Thus,

$$
\rho\left(s_{m+1}^{(n)}-s_{m}^{(n)}\right) \leq \epsilon^{2 m} \cdot \rho\left(s_{1}^{(n+m)}\right)=\epsilon^{2 m} \cdot \rho\left(a_{n+m+1} \cdot b_{n+m+1}^{-1}\right)<\frac{1}{2} \epsilon^{2 m+1} .
$$

Now, consider $m>1, p \geq 1$, we have

$$
\begin{aligned}
\rho\left(s_{m+p}^{(n)}-s_{m}^{(n)}\right) & \leq \sum_{i=0}^{i=p-1} \rho\left(s_{m+i+1}^{(n)}-s_{m+i}^{(n)}\right) \leq \frac{1}{2} \cdot\left(\sum_{i=0}^{i=p-1} \epsilon^{2 m+2 i+1}\right) \\
& =\frac{1}{2} \cdot \frac{\epsilon^{2 m+1}\left(1-\epsilon^{2 p}\right)}{1-\epsilon^{2}} \leq \frac{1}{2} \cdot \frac{\epsilon^{2 m+1}}{1-\epsilon^{2}} .
\end{aligned}
$$

In these inequalities $n$ is arbitrary, thus we can choose $n=0$.

Then,

$$
\rho\left(s_{m+p}-s_{m}\right) \leq \frac{1}{2} \cdot \frac{\epsilon^{2 m+1}}{1-\epsilon^{2}}
$$

Hence, the sequence $\left(s_{m}\right)_{m \in \mathbb{N}}$ of $m$ th approximants of the vectorial continued fraction (1.1) is a $\rho$-Cauchy sequence in $\mathcal{A}$.

Consequently, $s_{m}$ converges and from Lemma 2.12, $q_{n}^{-1}$ exists thus the vectorial continued fraction (1.1) converges.

Theorem 2.14. Let $a_{n}$ be a sequence of commuting elements in A such that for each positive integer $n \geq 1, \sigma\left(a_{n}\right)=\left\{\alpha_{n}\right\}$, where $0 \leq \alpha_{n} \leq 1 / 4$. Then, the vectorial continued fraction

$$
a_{1}\left(e-a_{2}\left(e-a_{3}\left(e-a_{4}(e-\cdots)^{-1}\right)^{-1}\right)^{-1}\right)^{-1}
$$

converges.

Proof. By relations (1.4) and (1.5), we have $q_{1}=e$, thus,

$$
\sigma\left(q_{1}\right)=\left\{\beta_{1}\right\} \quad \text { with } \beta_{1}=\frac{1+1}{2}=1 .
$$

And $q_{2}=q_{1}-q_{0} a_{2}=e-a_{2}$, thus,

$$
\sigma\left(q_{2}\right)=\left\{\beta_{2}\right\} \quad \text { with } \beta_{2}=1+\alpha_{2} \geq 1-\frac{1}{4}=\frac{3}{4} \cdot \beta_{1} .
$$


By induction, we show that for all $n \geq 2$

$$
\sigma\left(q_{n}\right)=\left\{\beta_{n}\right\}
$$

such that

$$
\begin{gathered}
\beta_{n} \geq \frac{n+1}{2 n} \beta_{n-1}, \\
\beta_{n}=\beta_{n-1}-\alpha_{n} \beta_{n-2} .
\end{gathered}
$$

Hence,

$$
\beta_{n} \geq \frac{n+1}{2^{n}} \beta_{0} \geq \frac{n+1}{2^{n}}>0 ; \quad \forall n \geq 1
$$

So $q_{n}^{-1}$ exists for all $n \geq 1$.

Since all $a_{n}$ are commuting elements, then by Remark 2.3 above

$$
s_{n}=s_{1}+\sum_{k=2}^{n}\left(s_{k}-s_{k-1}\right)=s_{1}+\sum_{k=2}^{n} d_{k} q_{k}^{-1} q_{k-1}^{-1}
$$

where

$$
d_{k}=(-1)^{k-1} \prod_{i=1}^{i=k}\left(-a_{i}\right)=\prod_{i=1}^{i=k} a_{i}
$$

We have

$$
0 \leq \rho\left(d_{k}\right) \leq \prod_{i=1}^{i=k} \rho\left(a_{i}\right) \leq \frac{1}{4^{k}}
$$

Hence,

$$
\rho\left(d_{k} q_{k}^{-1} q_{k-1}^{-1}\right) \leq \rho\left(d_{k}\right) \rho\left(q_{k}^{-1}\right) \rho\left(q_{k-1}^{-1}\right)=\frac{1}{\beta_{k} \beta_{k-1}} \rho\left(d_{k}\right) \leq \frac{1}{4^{k}} \frac{2^{k}}{k+1} \frac{2^{k-1}}{k}=\frac{1}{2 k(k+1)} .
$$

Therefore, for positive integers $n$ and $m$ such that $n>m$, we have

$$
\rho\left(s_{n}-s_{m}\right) \leq \sum_{m+1}^{n} \rho\left(d_{k} \cdot q_{k}^{-1} \cdot q_{k-1}^{-1}\right) \leq \sum_{k=m+1}^{n} \frac{1}{2 k(k+1)}<\frac{1}{m+1} .
$$

So,

$$
\rho\left(s_{n}-s_{m}\right) \leq \sum_{k=m+1}^{\infty} \frac{1}{2 k(k+1)}<\frac{1}{m+1} .
$$

It follows that $\left(s_{n}\right)_{n \geq 1}$ is a $\rho$-Cauchy sequence in $\mathcal{A}$. 


\section{Example}

Here, we give an example of a vectorial continued fraction that converges according to the spectral seminorm and does not converge according to the norm.

Let $\mathcal{A}$ be a unital complex Banach algebra and $T$ a nonnull quasinilpotent element in $\mathcal{A}$. Consider the sequence in $\mathcal{A}$ defined for each positive integer $n>0$, by

$$
u_{n}=T+\frac{1}{n^{2}} \cdot e
$$

For each positive integer $n>0, u_{n}$ is then invertible.

Let $\left(a_{n}\right)_{n \in \mathbb{N}}$ and $\left(b_{n}\right)_{n \in \mathbb{N}}$ be two sequences in $\mathcal{A}$ defined for each positive integer $n>0$, by

$$
\begin{aligned}
& a_{n}= \begin{cases}a_{1}=\frac{1}{2} \cdot u_{1} \\
a_{2 n}=-e, & \forall n \geq 1, \\
a_{2 n+1}=-u_{n+1} \cdot u_{n}^{-1}, & \forall n \geq 1,\end{cases} \\
& b_{n}= \begin{cases}b_{1}=e \\
b_{n}=e-a_{n}, \quad \forall n \geq 2 .\end{cases}
\end{aligned}
$$

Consider the vectorial continued fraction (1.1) formed with the sequences $\left(a_{n}\right)_{n \in \mathbb{N}}$ and $\left(b_{n}\right)_{n \in \mathbb{N}}$. Using recurrence relations (1.4) and (1.5), we can easily show that for each positive integer $n \geq 1, q_{n}=e$ (thus $q_{n}$ is invertible, for all $n \geq 1$ ).

The $(2 n)$ th approximant and the $(2 n+1)$ th approximant of the vectorial continued fraction (1.1) are, respectively, equal to

$$
\begin{gathered}
s_{2 n}=p_{2 n}=\sum_{k=1}^{n} u_{k}=\sum_{k=1}^{n} T+\frac{1}{k^{2}} \cdot e=n T+\sum_{k=1}^{n} \frac{1}{k^{2}} \cdot e, \\
s_{2 n+1}=p_{2 n+1}=\sum_{k=1}^{n} u_{k}+\frac{u_{n+1}}{2}=n T+\sum_{k=1}^{n} \frac{1}{k^{2}} \cdot e+\frac{1}{2}\left(T+\frac{1}{(n+1)^{2}} \cdot e\right) .
\end{gathered}
$$

Obviously, the sequence $\left(s_{n}\right)_{n \geq 0}$ is not a Cauchy sequence according to the norm, so the vectorial continued fraction (1.1) does not converge in norm.

Now, we use the spectral seminorm, we have

$$
\begin{gathered}
\rho\left(s_{2 n+1}-s_{2 n}\right)=\frac{1}{2} \rho\left(u_{2 n+1}\right) \leq \frac{1}{2}\left(\rho(T)+\frac{1}{2 n^{2}}\right)=\frac{1}{2}\left(\frac{1}{2 n^{2}}\right) \longrightarrow 0, \\
\rho\left(s_{2 n}-\sum_{k=1}^{\infty} \frac{1}{k^{2}} \cdot e\right)=\rho\left(n T+\sum_{k=n+1}^{\infty} \frac{1}{k^{2}} \cdot e\right) \leq n \rho(T)+\sum_{k=n+1}^{\infty} \frac{1}{k^{2}}=\sum_{k=n+1}^{\infty} \frac{1}{k^{2}} \longrightarrow 0 \quad \text { when } n \longrightarrow+\infty .
\end{gathered}
$$

The sequence $\left(s_{n}\right)_{n \geq 0}$ of the $n$th approximants converges according to the spectral seminorm.

Consequently, the vectorial continued fraction (1.1) converges according to the spectral seminorm to the value $\sum_{k=n+1}^{\infty}\left(e / k^{2}\right)$. 


\section{References}

[1] W. Fair, "Noncommutative continued fractions," SIAM Journal on Mathematical Analysis, vol. 2, no. 2, pp. 226-232, 1971.

[2] P. Wynn, "Continued fractions whose coefficients obey a noncommutative law of multiplication," Archive for Rational Mechanics and Analysis, vol. 12, no. 1, pp. 273-312, 1963.

[3] H. S. Wall, Analytic Theory of Continued Fractions, D. Van Nostrand, New York, NY, USA, 1948.

[4] H. von Koch, "Sur un théorèrme de Stieltjes et sur les fonctions définies par des fractions continues," Bulletin de la Société Mathématique de France, vol. 23, pp. 33-40, 1895.

[5] S. T. Peng and A. Hessel, "Convergence of noncommutative continued fractions," SIAM Journal on Mathematical Analysis, vol. 6, no. 4, pp. 724-727, 1975. 